\title{
On Bounding the Betti Numbers and Computing the Euler Characteristic of Semi-Algebraic Sets*
}

\author{
S. Basu \\ Department of Mathematics, University of Michigan, \\ Ann Arbor, MI 48109, USA \\ saugata@math.lsa.umich.edu
}

\begin{abstract}
In this paper we prove new bounds on the sum of the Betti numbers of closed semi-algebraic sets and also give the first single exponential time algorithm for computing the Euler characteristic of arbitrary closed semi-algebraic sets.

Given a closed semi-algebraic set $S \subset R^{k}$ defined as the intersection of a real variety, $Q=0, \operatorname{deg}(Q) \leq d$, whose real dimension is $k^{\prime}$, with a set defined by a quantifierfree Boolean formula with no negations with atoms of the form $P_{i}=0, P_{i} \geq 0, P_{i} \leq$ $0, \operatorname{deg}\left(P_{i}\right) \leq d, 1 \leq i \leq s$, we prove that the sum of the Betti numbers of $S$ is bounded by $s^{k^{\prime}}(O(d))^{k}$. This result generalizes the Oleinik-Petrovsky-Thom-Milnor bound in two directions. Firstly, our bound applies to arbitrary unions of basic closed semi-algebraic sets, not just for basic semi-algebraic sets. Secondly, the combinatorial part (the part depending on $s$ ) in our bound, depends on the dimension of the variety rather than that of the ambient space. It also generalizes the result in [4] where a similar bound is proven for the number of connected components. We also prove that the sum of the Betti numbers of $S$ is bounded by $s^{k^{\prime}} 2^{O\left(k^{2} m^{4}\right)}$ in case the total number of monomials occurring in the polynomials in $\mathcal{P} \cup\{Q\}$ is $m$. Using the tools developed for the above results, as well as some additional techniques, we give the first single exponential time algorithm for computing the Euler characteristic of arbitrary closed semi-algebraic sets.
\end{abstract}

\section{Introduction}

Let $\mathcal{P}=\left\{P_{1}, \ldots, P_{s}\right\} \subset R\left[X_{1}, \ldots, X_{k}\right]$ be a family of polynomials whose degrees are bounded by $d$ and $S$ is a closed semi-algebraic set defined by a quantifier-free Boolean

\footnotetext{
* This work was done while the author was a graduate student at the Courant Institute of Mathematical Sciences, New York University, New York, NY 10012, USA, and was supported in part by NSF Grants CCR9402640 and CCR-9424398. A preliminary version of this paper appeared in the Proceedings of the 28th Annual ACM Symposium on the Theory of Computing, pp. 408-417, 1996.
} 
formula without negations, whose atoms are of the form $P_{i} \sigma 0$ where $\sigma \in\{\geq, \leq,=\}$ for $1<i<s$.

Thus $S$ is defined by a disjunction of weak sign conditions on the family $\mathcal{P}$, where a weak sign condition is a conjunction of the form

$$
P_{1} \sigma_{1} 0, \ldots, P_{s} \sigma_{s} 0 \quad \text { where } \quad \sigma_{i} \in\{\geq, \leq,=\} \quad \text { for } \quad 1 \leq i \leq s .
$$

The Betti numbers $\beta_{i}(S)$, the ranks of the singular homology groups of $S$, are a measure of the topological complexity of $S$ and can be bounded in terms of $s, d$, and $k$ as follows. Collins' algorithm [9] for cylindrical algebraic decomposition gives a cellular decomposition of $S$ into $(s d)^{2^{O(k)}}$ cells and thus the same bound applies to the $\beta_{i}(S)$. In case $S$ is a basic closed semi-algebraic set defined by $P_{1} \geq 0, \ldots, P_{s} \geq 0$, with $\operatorname{deg}\left(P_{i}\right) \leq d$, the tighter bound of $(O(s d))^{k}$ on the sum of the Betti numbers of $S$ was proved in separate papers by Oleinik and Petrovsky [25], Thom [29], and Milnor [23]. (Note that when $S$ is the set of real zeros of a set of polynomials this bound can be reduced to $(O(d))^{k}$.) Similar bounds on the number of connected components of basic semi-algebraic sets were also proved by Warren [30]. These bounds play an important role in algorithmic real algebraic geometry [17], in discrete geometry [12], [13], and have been used recently in proving lower bounds in the algebraic computation tree model (see [31] and [24]).

A difficulty in extending this bound to an arbitrary semi-algebraic set defined by the polynomials $\mathcal{P}$, lies in the fact that the Betti numbers of the union of two disjoint sets can be arbitrarily greater than the sum of the individual Betti numbers of the two sets (see [31] for an easy example).

We extend this bound to arbitrary unions of basic closed semi-algebraic sets by proving the following theorem:

Theorem 1. Let $S \subset R^{k}$ be the intersection of a closed semi-algebraic set defined by a quantifier-free Boolean formula without negations involving a family $\mathcal{P}=\left\{P_{1}, \ldots, P_{s}\right\}$, of s polynomials whose atoms are of the form $P_{i} \sigma 0, \sigma \in\{\geq, \leq,=\}$ with $Z(Q)$, the zero set of a polynomial $Q$. If the geometric dimension of $Z(Q)$ is $k^{\prime}$, and the degrees of the polynomials in $\mathcal{P} \cup\{Q\}$ are all bounded by $d$. Then the sum of the Betti numbers of $S$ is bounded by $s^{k^{\prime}}(O(d))^{k}$.

If $S$ can be more simply described as $Q=0, P_{1}>0, \ldots, P_{s}>0$, then we have the slightly better bound of $\left(\begin{array}{l}s \\ k^{\prime}\end{array}\right)(O(d))^{k}$ on the sum of the Betti numbers of $S$.

If $Q$ is the zero polynomial, then $Z(Q)=R^{k}, k^{\prime}=k$, and we obtain the OleinikPetrovsky-Thom-Milnor bound extended to arbitrary closed semi-algebraic sets as a special case. Note that a bound of $\left(\begin{array}{c}s \\ k^{\prime}\end{array}\right)(O(d))^{k}$ on the zeroth Betti number for arbitrary semi-algebraic sets (which is just the number of connected components) was known before (see [4]) and our bound is a generalization of this result to the sum of the Betti numbers in the case of closed semi-algebraic sets.

Note also that a lower bound of $\left(s d / k^{\prime}\right)^{k^{\prime}}$ on the zeroth Betti number is easily obtained by considering the set of nonzeros of $s$ polynomials, each of them a product of $d$ linear polynomials, restricted to a $k^{\prime}$ dimensional linear subspace.

The dependence of the combinatorial part (the part depending on $s$ ) of the bound in Theorem 1 on $k^{\prime}$ instead of $k$ is important when we consider low-dimensional semialgebraic sets embedded in a higher-dimensional space, and this is sometimes important 
in applications. For example, the bound on the number of connected components in [4] plays a crucial role in the proof of the main result in [13], where the variety is the real Grassmannian $G_{m, n}$ (the space of $m$-dimensional subspaces of $R^{n}$ ), embedded as an $m(n-m)$-dimensional variety in $R^{n^{2}}$.

Remark 1. We note that a bound of $(O(s d))^{2 k}$ on the sum of the Betti numbers of compact semi-algebraic sets can be deduced from Theorem 1 in [31] or from Theorem 1.10 in [24]. Also note that recently Barvinok [1] has proved a new bound on the sum of Betti numbers of semi-algebraic sets defined by quadratic inequalities which is polynomial in the number of variables when the number of inequalities is constant.

Remark 2. Even though we state our results for closed semi-algebraic sets over the reals the same bounds hold over any real closed field through the application of wellknown transfer principles. We refer the reader to the article by Knebusch [21] and to [6] for the intricacies of semi-algebraic topology over general real closed fields.

In order to achieve the bound in Theorem 1 we prove that an arbitrary closed semialgebraic set has the same homology groups as a compact semi-algebraic set defined by polynomials in general position. This result generalizes a similar result in [7] where it was proved for semi-algebraic sets defined by a single sign condition, and this intermediate result might be of independent interest.

Next we consider the problem of computing the Euler characteristic, $\chi(S)$, of a closed semi-algebraic set $S$. The Euler characteristic, which is the alternating sum of the Betti numbers of $S$, is an important topological invariant and thus can be used as a test to rule out topological equivalence. Also, computing the Euler characteristic of semi-algebraic sets occurs as an important subproblem in some recent work due to Gabrielov [11] on computing multiplicities of the zeros of polynomial functions along the trajectories of a polynomial vector field.

We prove the following theorem.

Theorem 2. Let $S \subset R^{k}$ be a real closed semi-algebraic set defined by a quantifier-free Boolean formula without negation, with atoms of the form $P_{i} \sigma 0$ where $\sigma \in\{\geq, \leq,=\}$ for $1 \leq i \leq s$, where $\mathcal{P}=\left\{P_{1}, \ldots, P_{s}\right\} \subset Z\left[X_{1}, \ldots, X_{k}\right]$, is a family of $s$ polynomials whose degrees are bounded by $d$, and the bit lengths of the coefficients of $P_{i}$ are bounded by $L$. Then there exists an algorithm for computing $\chi(S)$ which performs at most $(k s d)^{O(k)} L^{O(1)}$ bit operations.

We remark that computing the homology groups of semi-algebraic sets in single exponential time is a central open problem of computational real algebraic geometry. Single exponential algorithms for determining certain other (weaker) topological properties of semi-algebraic sets are known. For example, it is possible to compute the number of connected components [15], [7], [14], [18], the semi-algebraic description of the connected components [8], [19], as well as to decide whether two points are in the same connected component of a semi-algebraic set [7], [5], in single exponential time. 
Collins' algorithm for computing a cylindrical algebraic decomposition [9] gives sufficient topological information for computing the Euler characteristic, and in fact the homology groups of a given semi-algebraic set [27]. However, this algorithm has double exponential complexity $(s d)^{2^{O(k)}} L^{O(1)}$ where $L$ is a bound on the number of bits used in specifying the coefficients of the input polynomials. Previously, this was the best algorithm for computing the Euler characteristic of general semi-algebraic sets. A single exponential algorithm for computing the Euler characteristic of a smooth algebraic hypersurface is mentioned in [26].

The rest of the paper is organized as follows. In Section 2 we show how to perturb the polynomials to bring them into general position without changing the homology groups of the given semi-algebraic set. In Section 3 we prove our bound on the Betti numbers of closed semi-algebraic sets. In Section 4 we prove a bound on the sum of the Betti numbers of a closed semi-algebraic set, in which the algebraic part of the complexity depends only on the number of monomials appearing in the polynomials and is independent of the degree. In Section 5 we describe our algorithm for computing the Euler characteristic. In Section 5.2 we give an algorithm for computing the Euler characteristic of a basic semi-algebraic set, and in Section 5.3 we give an algorithm for computing the Euler characteristic of an arbitrary union of such sets.

\section{Going to General Position}

In the proofs of our theorems as well as in the algorithms to be described later we often make use of perturbations by infinitesimals. For the purposes of this paper it is enough to consider an infinitesimal as a sufficiently small positive real number. We also use the notation $\varepsilon_{1} \gg \varepsilon_{2} \gg \varepsilon_{3} \cdots$, where $\varepsilon_{1}, \varepsilon_{2}, \varepsilon_{3}$ are infinitesimals and $\varepsilon_{1}$ is positive and sufficiently small, $\varepsilon_{2}$ is positive and sufficiently small with respect to $\varepsilon_{1}$, and so on.

\subsection{General Position}

We say that a family of polynomials $\mathcal{P}$ in $k$ variables is in general position if no $k+1$ of them have a common real zero. In this section we show that given a semi-algebraic set, $S$, defined by a family, $\mathcal{P}$, of $s$ polynomials with degrees bounded by $d$, we can define a new compact semi-algebraic set $S^{\prime}$, which has the same homology groups as $S$, but which is defined by a family, $\mathcal{P}^{\prime}$, of polynomials in general position. Moreover, $\left|\mathcal{P}^{\prime}\right| \leq 4 s+1$, and the degrees of the polynomials in $\mathcal{P}^{\prime}$ are bounded by $d^{\prime}$, where $d^{\prime}$ is the least even number greater than $d$. Similar results appear in [7], where they are proved for semi-algebraic sets defined by one single sign condition. Our proof techniques are similar to those used in [7], but our results apply to arbitrary closed semi-algebraic sets.

We first recall a few facts from algebraic topology. Given a semi-algebraic set, $S \subset R^{k}$, we denote by $H_{*}(S)$ the graded singular homology group of $S$.

The following theorem appears in [28] (Theorem 4.1.7).

Theorem 3. The singular homology functor commutes with direct limits. 
We also need the following fundamental property of the $\check{C} e c h$ homology groups of a compact space (see p. 257 of [10]).

Theorem 4. If $X$ is compact and the inverse limit of compact sets $X_{\alpha}$, then the $\check{C}$ ech homology group $H_{*}(X)$ is the inverse limit of the $\check{C}$ ech homology groups $H_{*}\left(X_{\alpha}\right)$.

Note that, since compact semi-algebraic sets are triangulable, their singular and $\check{C} e c h$ homologies coincide.

We first ensure that a given semi-algebraic set has the same homology groups as a bounded one.

Lemma 1. Let $S$ be any closed semi-algebraic set. Let $S_{\Omega}=S \cap\left(X_{1}^{2}+\cdots+X_{k}^{2} \leq \Omega\right)$. Then, for sufficiently large $\Omega, H_{*}(S) \cong H_{*}\left(S_{\Omega}\right)$.

Proof. Let $S^{\prime} \subset R^{k+1}$ be the set defined by the same formula as $S$ plus the new inequality $T\left(X_{1}^{2}+\cdots+X_{k}^{2}\right) \leq 1$. Let $\pi$ and $\pi_{x}$ denote the projections onto the $T$ and the $X$ coordinates, respectively. Then, by Hardt's triviality theorem [16], for all sufficiently large $\Omega$, and for all $t \in(0,1 / \Omega), S^{\prime} \cap \pi^{-1}(t)$ is homeomorphic to $S^{\prime} \cap \pi^{-1}(1 / \Omega)$.

Moreover, each $S^{\prime} \cap \pi^{-1}(t)$ is compact and $S=\bigcup_{t>0} \pi_{x}\left(S^{\prime} \cap \pi^{-1}(t)\right)$. It is also clear that the singular chain complex of $S$ is the direct limit of the singular chain complexes of $\pi_{x}\left(S^{\prime} \cap \pi^{-1}(t)\right)$. Now, by Theorem 3, $H_{*}(S)$ is the direct limit of the groups $H_{*}\left(\left(S^{\prime} \cap\right.\right.$ $\left.\left.\pi^{-1}(t)\right)\right)$.

Hence, $H_{*}(S) \cong H_{*}\left(S^{\prime} \cap \pi^{-1}(1 / \Omega)\right) \cong H_{*}\left(S_{\Omega}\right)$.

Let $S$ be a compact semi-algebraic set defined by the formula

$$
\left(\bigvee_{1 \leq j \leq L} \sigma_{j}(\mathcal{P})\right) \wedge\left(X_{1}^{2}+\cdots+X_{k}^{2} \leq \Omega\right)
$$

where each $\sigma_{j}$ is a nonempty weak sign condition for the family $\mathcal{P}$, and $\Omega$ is positive and sufficiently large.

For any weak sign condition $\sigma$ and a new variable $\varepsilon$ we denote by $\sigma(\mathcal{P}, \varepsilon)$ the following formula: For every weak inequality in $\sigma$ we replace the corresponding conjunct $P_{j} \geq 0$ (resp. $P_{j} \leq 0$ ) by $P_{j}>-\varepsilon$ (resp. $P_{j}<\varepsilon$ ). For every equality in $\sigma$ we replace the corresponding conjunct $P_{j}=0$ by the conjunct $\left(P_{j}>-\varepsilon\right) \wedge\left(P_{j}<\varepsilon\right)$.

Let $S_{\varepsilon}$ denote the semi-algebraic set defined by the formula $\bigvee_{1 \leq j \leq L} \sigma_{j}(\mathcal{P}, \varepsilon)$.

We have the following lemma.

Lemma 2. Let $S$ be compact and let $S_{\varepsilon}$ be as above. Then, for sufficiently small $\varepsilon>0$, $H_{*}(S) \cong H_{*}\left(S_{\varepsilon}\right)$.

Proof. Let $S_{\varepsilon}^{\prime}$ denote the semi-algebraic set defined as $S_{\varepsilon}$ with the difference that every inequality is replaced by the corresponding weaker version and the constraint $X_{1}^{2}+\cdots+X_{k}^{2} \leq R$ is added, where $R$ is the radius of a ball containing $S$. It follows that 
$S_{\varepsilon}^{\prime}$ is compact. Again by Hardt's triviality theorem, for sufficiently small $\varepsilon>0$, and all $0<\delta<\varepsilon, S_{\delta}^{\prime}$ is homeomorphic to $S_{\varepsilon}^{\prime}$.

Now, $S \subset S_{\varepsilon} \subset S_{\varepsilon}^{\prime}$. Also, $S=\bigcap_{\varepsilon>0} S_{\varepsilon}^{\prime}$. Hence, by Theorem 4, $H_{*}(S)$ is the inverse limit of the groups $H_{*}\left(S_{\varepsilon}^{\prime}\right)$, and thus by the previous observation $H_{*}(S) \cong H_{*}\left(S_{\varepsilon}^{\prime}\right)$. Finally, note that $S_{\varepsilon}=\bigcup_{0<t<\varepsilon} S_{t}^{\prime}$, and the singular chain complex of $S_{\varepsilon}$ is the direct limit of the singular chain complexes of $S_{t}^{\prime}$.

Thus, by Theorem $3, H_{*}\left(S_{\varepsilon}\right)$ is the direct limit of the groups $H_{*}\left(S_{t}^{\prime}\right)$. However, $H_{*}\left(S_{t}^{\prime}\right) \cong H_{*}\left(S_{\varepsilon}^{\prime}\right)$ for $0<t<\varepsilon$. Thus, we have that $H_{*}\left(S_{\varepsilon}\right) \cong H_{*}\left(S_{\varepsilon}^{\prime}\right)$ which implies that $H_{*}(S) \cong H_{*}\left(S_{\varepsilon}\right)$.

Note that the set $S_{\varepsilon}$ is defined by a disjunction of conjunctions of strict inequalities involving $2 s$ polynomials,

$$
\bigcup_{P \in \mathcal{P}}\{P+\varepsilon, P-\varepsilon\}
$$

and that there are no equalities in the formula.

We next prove that given a semi-algebraic set defined by a disjunct of conjuncts of the form $\bigwedge_{j} Q_{j} s_{j} 0$ where $s_{j} \in\{<,>\}$, it is then possible to define a new semi-algebraic set defined by polynomials in general position having the same homology group.

Let $S$ be a semi-algebraic set defined by the formula $\bigvee_{1 \leq j \leq L} \sigma_{j}\left(\mathcal{Q}_{j}\right)$, where each $\sigma_{j}$ is a sign-condition on a subset $\mathcal{Q}_{j}$ of a family of polynomial $\mathcal{Q}$, and $\sigma_{j}$ does not contain equality. Let $\mathcal{Q}=\left\{Q_{1}, \ldots, Q_{s}\right\}$ and $\operatorname{deg}\left(Q_{i}\right)<d, 1 \leq i \leq s$.

For $1 \leq i \leq 2 s$, let $H_{i}=1+\sum_{1 \leq j \leq k} i^{j} X_{j}^{d^{\prime}}$, where $\bar{d}^{\prime}$ is the least even number larger than $d$. For $Q_{i} \in \mathcal{Q}$, let $Q_{i}^{+}=(1-\delta) Q_{i}+\delta H_{2 i-1}$ and $Q_{i}^{-}=(1-\delta) Q_{i}-\delta H_{2 i}$, where $\delta$ is a new variable. Let $\mathcal{Q}^{\prime}$ be the family $\bigcup_{i}\left\{Q_{i}^{+}, Q_{i}^{-}\right\}$.

Lemma 3. For sufficiently small $\delta>0$, the family of polynomials $\mathcal{Q}^{\prime}$ is in general position.

Proof. See [3].

For a sign condition $\sigma$ on $\mathcal{Q}$, without any equalities, define $\sigma(\mathcal{Q}, \delta)$ to be the formula obtained as follows:

Every conjunct $Q_{i}>0$ is replaced by $Q_{i}^{-} \geq 0$, while every conjunct $Q_{i}<0$ is replaced by $Q_{i}^{+} \leq 0$.

Let $S_{\delta}^{-}$be the set defined by $\bigvee_{1 \leq j \leq L} \sigma_{j}\left(\mathcal{Q}_{j}, \delta\right)$.

Lemma 4. Let $S$ and $S_{\delta}^{-}$be as above. Then, for sufficiently small $\delta>0, H_{*}\left(S_{\delta}^{-}\right) \cong$ $H_{*}(S)$.

Proof. Again by Hardt's triviality theorem, $H_{*}\left(S_{t}^{-}\right)$are isomorphic for all $t \in(0, \delta)$ for sufficiently small $\delta$. Also, $S=\bigcup_{0<t} S_{t}^{-}$, and the singular chain complex of $S$ is the direct limit of the singular chain complexes of $S_{t}^{-}$. Again applying Theorem 3 we have that $H_{*}(S) \cong H_{*}\left(S_{\delta}^{-}\right)$, for all sufficiently small $\delta>0$.

Combining the previous four lemmas we obtain the following proposition. 
Proposition 1. Let $S$ be any semi-algebraic set defined by a family of $s$ polynomials with degrees bounded by $d$. Then there exists a set $S(\Omega, \varepsilon, \delta)$ defined by a family of at most $4 s+1$ polynomials, in $X_{1}, \ldots, X_{k}, \varepsilon, \delta, \Omega$ with degrees bounded by $2 d$ such that, for sufficiently small $1 / \Omega \gg \varepsilon \gg \delta>0$, this new family of polynomials is in general position and $H_{*}(S(\Omega, \varepsilon, \delta)) \cong H_{*}(S)$. Moreover, the set $S(\Omega, \varepsilon, \delta)$ is compact, and defined as a disjunction of conjunctions of weak inequalities.

In our proofs we need a slightly stronger notion of general position.

Let $Q$ be a polynomial such that $Z(Q)$ has geometric dimension $k^{\prime}$. We say that a family of polynomials $\mathcal{P}$ is in general position with respect to $Q$, if no $k^{\prime}+1$ of the polynomials in $\mathcal{P}$ have a real zero in common with $Q$.

We need the following proposition whose proof appears in [4].

Proposition 2. Given a family $\left\{P_{1}, \ldots, P_{s}\right\}$ of polynomials in $R\left[X_{1}, \ldots, X_{k}\right]$ and a variety $Z(Q)$ of real dimension $k^{\prime}$ and infinitesimals $\delta_{1} \gg \cdots \gg \delta_{s} \gg \delta$, the perturbed family $\mathcal{P}^{*}=\bigcup_{1 \leq i \leq s}\left\{P_{i}-\delta_{i}, P_{i}+\delta_{i}, P_{i}-\delta \delta_{i}, P_{i}+\delta \delta_{i}\right\}$ is in general position with respect to the variety $Z(Q)$.

Using the above proposition we have the following more general version of Proposition 1.

Proposition 3. Let $S$ be any semi-algebraic set defined by a family of s polynomials with degrees bounded by $d$ and contained in a real variety $Z(Q)$ of real dimension $k^{\prime}$. Then there exists a set $S\left(\Omega, \varepsilon, \delta_{1}, \ldots, \delta_{s}, \delta\right)$ defined by a family of at most $4 s+1$ polynomials, in $X_{1}, \ldots, X_{k}, \varepsilon, \delta, \delta_{1}, \ldots, \delta_{s}, \Omega$ with degrees bounded by $2 d$ such that, for sufficiently small $1 / \Omega \gg \varepsilon \gg \delta_{1} \gg \cdots \gg \delta_{s} \gg \delta>0$, this new family of polynomials is in general position with respect to $Q$, and $H_{*}\left(S\left(\Omega, \varepsilon, \delta_{1}, \ldots, \delta_{s}, \delta\right)\right) \cong H_{*}(S)$. Moreover, the set $S\left(\Omega, \varepsilon, \delta_{1}, \ldots, \delta_{s}, \delta\right)$ is compact, and defined as a disjunction of conjunctions of weak inequalities.

Proof. The proof is the same as that of Proposition 1, always carrying along the extra condition $Q=0$, and replacing the polynomials $H_{i}$ by the infinitesimals $\delta_{i}$.

We also remark that if a family of polynomials $\mathcal{P}$ is in general position with respect to a polynomial $Q$, then for an infinitesimal $\varepsilon$ the family $\bigcup_{P \in \mathcal{P}}\{P, P+\varepsilon, P-\varepsilon\}$ is also in general position with respect to $Q$ as long as we consider zeros that are bounded over $R$.

\section{Bounding the Betti Numbers}

We make use of the following facts from algebraic topology which follows easily from the Mayer-Vietoris sequence (see [28]). Given a semi-algebraic set $S$ we define $r(S)=$ $\sum_{i} \beta_{i}(S)$. Let $S_{1}$ and $S_{2}$ be two compact semi-algebraic sets. Then, from the Mayer- 
Vietoris sequence

$$
\begin{aligned}
\cdots \rightarrow H_{i+1}\left(S_{1} \cup S_{2}\right) & \rightarrow H_{i}\left(S_{1} \cap S_{2}\right) \rightarrow H_{i}\left(S_{1}\right) \oplus H_{i}\left(S_{2}\right) \\
& \rightarrow H_{i}\left(S_{1} \cup S_{2}\right) \rightarrow H_{i-1}\left(S_{1} \cap S_{2}\right) \rightarrow \cdots,
\end{aligned}
$$

it easily follows that

$$
r\left(S_{1}\right)+r\left(S_{2}\right) \leq r\left(S_{1} \cup S_{2}\right)+r\left(S_{1} \cap S_{2}\right)
$$

and

$$
r\left(S_{1} \cup S_{2}\right) \leq r\left(S_{1}\right)+r\left(S_{2}\right)+r\left(S_{1} \cap S_{2}\right)
$$

Lemma 5. Let $S$ be a semi-algebraic set defined by a conjunct $(Q=0) \wedge\left(\sigma_{1}(\mathcal{P}) \vee\right.$ $\cdots \vee \sigma_{L}(\mathcal{P})$ ), where $Q$ is a polynomial, and $\sigma_{j}, 1 \leq j \leq L$, are distinct sign conditions on a family of polynomials $\mathcal{P}$, such that none of the $\sigma_{j}$ contain an equality. Then $\beta_{i}(S)=$ $\sum_{1 \leq j \leq L} \beta_{i}\left(S_{j}\right)$ and $\chi(S)=\sum_{1 \leq j \leq L} \chi\left(S_{j}\right)$, where $S_{j}$ is the set defined by $(Q=0) \wedge$ $\sigma_{j}(\mathcal{P})$.

Proof. This follows immediately from the definition of singular homology groups. The singular chain of $S$ being the direct sum of the singular chains of the $S_{j}$ 's.

\subsection{Proof of Theorem 1}

Proof. In view of Proposition 3 and the remarks following it we can assume without loss of generality that $S$ is a compact set defined by $s$ polynomials in general position with respect to $Q$.

We next prove two lemmas that will imply the theorem.

Given a polynomial $Q$ and a family of polynomials $\mathcal{P}=\left\{P_{1}, \ldots, P_{s}\right\}$, we define the combinatorial level of the system $(Q, \mathcal{P})$ to be the least integer $m$ such that no $m+1$ of the polynomials in $\mathcal{P}$ have a common real zero with $Q$.

For example, the combinatorial level of $(Q, \mathcal{P})$ is bounded by $k^{\prime}$ if the dimension of $Z(Q)$ is $k^{\prime}$ and the polynomials in $\mathcal{P}$ are in general position with respect to $Q$.

Lemma 6. Let $S$ be a semi-algebraic set, defined by $Q=0, P_{1}>0, \ldots, P_{s}>0$, where $Z(Q)$ is bounded and $Q$ is nonnegative everywhere. Let $\mathcal{P}=\left\{P_{1}, \ldots, P_{s}\right\}$, and let the combinatorial level of the system $(Q, \mathcal{P})$ be bounded by $m \leq k$ and let the degrees of the polynomials $Q$ and $P_{i}$ be bounded by $2 d$ and $d$, respectively. Then $r(S)=\left(\begin{array}{c}s \\ m\end{array}\right)(O(d))^{k}$.

Proof. Consider the set $S_{\varepsilon}$ defined by

$$
Q=0, P_{1} \geq \varepsilon, \ldots, P_{s} \geq \varepsilon
$$

where $\varepsilon>0$ is a positive infinitesimal. Then it is easy to prove that, for sufficiently small $\varepsilon>0, H_{*}\left(S_{\varepsilon}\right) \cong H_{*}(S)$. Let $T$ denote the set defined by $Q=0, P_{2} \geq \varepsilon, \ldots, P_{s} \geq \varepsilon$. 
Consider the sets $U_{1}=T \cap\left(\left(P_{1} \geq \varepsilon\right) \cup\left(P_{1} \leq \varepsilon\right)\right), V_{1}=T \cap\left(-\varepsilon \leq P_{1} \leq \varepsilon\right)$ and $W_{1}=T \cap\left(\left(P_{1}=\varepsilon\right) \cup\left(P_{1}=-\varepsilon\right)\right)$.

From inequality (1) we see that $r\left(U_{1}\right)+r\left(V_{1}\right) \leq r(T)+r\left(W_{1}\right)$ and from Lemma 5 that $r(S) \leq r\left(U_{1}\right)$. It immediately follows that $r(S) \leq r(T)+r\left(W_{1}\right)$.

Moreover, let $r(s, d, m, k)$ be the maximum possible value of $r(S)$ for any set defined by a system with these parameters. Then we have the recurrence,

$$
r(s, d, m, k) \leq r(s-1, d, m, k)+2 r(s-1, d, m-1, k), \quad m \leq k .
$$

Using the Oleinik-Petrovsky-Thom-Milnor bound for algebraic sets, we have

$$
r(0, d, m, k)=(O(d))^{k}, \quad r(s, d, 0, k)=(O(d))^{k} .
$$

It follows easily that $r(s, d, m, k)=\left(\begin{array}{c}s \\ m\end{array}\right)(O(d))^{k}$.

Lemma 7. Let $S$ be a compact semi-algebraic set contained in the zero set of a polynomial $Q$ and defined by a family of polynomials $\mathcal{P}=\left\{P_{1}, \ldots, P_{s}\right\}$. Suppose that $Q$ is nonnegative everywhere, the combinatorial level of the system $(Q, \mathcal{P})$ is bounded by $m \leq k$, and the degrees of the polynomials $Q$ and $P_{i}$ are bounded by $2 d$ and $d$, respectively. Then $r(S)=s^{m}(O(d))^{k}$.

Proof. Let $S$ be defined by the conjunct $(Q=0) \wedge\left(\sigma_{1} \vee \cdots \wedge \sigma_{L}\right)$ where the $\sigma_{i}$ are sign conditions on the family $\mathcal{P}$. Let $\varepsilon_{1} \gg \varepsilon_{2} \gg \cdots \gg \varepsilon_{s}>0$ be infinitesimals. Consider the sets $T_{1}, U_{1}, V_{1}, W_{1}^{\prime}, W_{1}$ defined as follows:

$$
\begin{aligned}
T_{1} & =S \cap\left(\left(P_{1} \geq \varepsilon_{1}\right) \cup\left(P_{1} \leq-\varepsilon_{1}\right)\right), \\
U_{1} & =S \cap\left(P_{1}=\varepsilon_{1}\right), \\
V_{1} & =S \cap\left(P_{1}=-\varepsilon_{1}\right), \\
W_{1}^{\prime} & =S \cap\left(-\varepsilon_{1} \leq P_{1} \leq \varepsilon_{1}\right),
\end{aligned}
$$

and

$$
W_{1}=S \cap\left(P_{1}=0\right) .
$$

Now $S=T_{1} \cup W_{1}^{\prime}$, and it is clear that $T_{1} \cap W_{1}^{\prime}=U_{1} \cup V_{1}$ and $U_{1} \cap V_{1}=\emptyset$.

Using inequality (2) twice, along with the fact that $r(\emptyset)=0$, we have

$$
r(S) \leq r\left(T_{1}\right)+r\left(W_{1}^{\prime}\right)+r\left(U_{1}\right)+r\left(V_{1}\right) .
$$

Moreover, using the same arguments as in Lemmas 2 and 4, it can be shown that $H_{*}\left(W_{1}^{\prime}\right) \cong H_{*}\left(W_{1}\right)$. We omit the proof as the arguments are completely analogous.

Thus, we have

$$
r(S) \leq r\left(T_{1}\right)+r\left(W_{1}\right)+r\left(U_{1}\right)+r\left(V_{1}\right) .
$$

Note that $U_{1}, V_{1}, W_{1}$ are defined by the systems $\left(Q+\left(P_{1}-\varepsilon_{1}\right)^{2}, \mathcal{P}\right),\left(Q+\left(P_{1}+\varepsilon_{1}\right)^{2}, \mathcal{P}\right)$, $\left(Q+P_{1}^{2}, \mathcal{P} \backslash\left\{P_{1}\right\}\right)$ (note that $Q$ is nonnegative everywhere), respectively. Moreover, each of the above system has a combinatorial level at most $m-1$. 
We next consider $T_{1}$ which is defined by a set of sign conditions without the atom $P_{1}=0$, and eliminate the atom $P_{2}=0$. We do this by replacing $P_{2}<0, P_{2}>0$ by $P_{2} \leq-\varepsilon_{2}, P_{2} \geq \varepsilon_{2}$, respectively. In this way we obtain the inequality

$$
r\left(T_{1}\right) \leq r\left(T_{2}\right)+r\left(W_{2}\right)+r\left(U_{2}\right)+r\left(V_{2}\right),
$$

where $U_{2}, V_{2}, W_{2}$ are sets defined by systems with a combinatorial level at most $m-1$.

The remaining set, $T_{2}$, has the same homology as the union of the sets defined by those sign conditions appearing in the definition of $S$, which contain neither $P_{1}=0$ nor $P_{2}=0$.

We continue this process till we have eliminated $P_{s}=0$, and we get the inequality

$$
r(S) \leq r\left(T_{s}\right)+\sum_{1 \leq i \leq s}\left(r\left(U_{i}\right)+r\left(V_{i}\right)+r\left(W_{i}\right)\right) .
$$

The sets $U_{i}, V_{i}, W_{i}$ are defined by systems of at most $s$ polynomials having a combinatorial level at most $m-1$. Moreover, the remaining term $r\left(T_{s}\right)$ is the bound on the Betti numbers of a semi-algebraic set defined by a union of sign conditions of the form $Q=0, P_{i} s_{i} 0$ for $1 \leq i \leq s$, with $s_{i} \in\{<,>\}$.

Again, by Lemma 5, the Betti numbers of this set are the sum of the the Betti numbers of the nonempty sets defined by each individual sign condition. Now consider the set $T$ defined by $Q=0, P_{1}^{2}>0, \ldots, P_{s}^{2}>0$. From the above remark it is clear that $r\left(T_{s}\right) \leq$ $r(T)$. Moreover, applying the bound proved in Lemma 6 we have $r(T)=\left(\begin{array}{c}s \\ m\end{array}\right)(O(d))^{k}$.

Let $r(s, d, k, m)$ denote the sum of the Betti numbers of a semi-algebraic set defined by a system $(Q, \mathcal{P})$ with $|\mathcal{P}|=s$, a combinatorial level of the system bounded by $m$, and the degrees of the polynomial $Q$ and those in $\mathcal{P}$ bounded by $d$. Then we have the recurrence

$$
\begin{gathered}
r(s, d, k, m)=\left(\begin{array}{c}
s \\
m
\end{array}\right)(O(d))^{k}+3 \operatorname{sr}(s, d, k, m-1), \quad m \leq k, \\
r(s, d, k, 0)=(O(d))^{k} .
\end{gathered}
$$

This recurrence solves to $r(s, d, k, m)=s^{m}(O(d))^{k}, m \leq k$, which proves the lemma.

The theorem now follows since the combinatorial level of a system $(Q, \mathcal{P})$ with the family $\mathcal{P}$ in general position with respect to $Q$ is bounded by $k^{\prime}$.

\section{A Fewnomial Bound}

In this section we prove a bound on the sum of the Betti numbers of a closed semialgebraic set, in which the algebraic part of the complexity depends only on the number of monomials appearing in the polynomials and is independent of the degree. This result is a consequence of the fewnomial bounds due to Khovansky [20] and the technique used to prove Theorem 1 . 
The following proposition is crucial for the proving the bound and is used as well in our algorithms to compute the Euler characteristic. Let $S$ be a compact, basic, semialgebraic set defined by $P_{1} \geq 0, \ldots, P_{S} \geq 0$. The next proposition proves that $S$ has the same homology groups as a certain set which is bounded by a smooth hypersurface.

Proposition 4. Let $Q=\prod_{1 \leq i \leq s}\left(\zeta+(1-\zeta) P_{i}\right)-\zeta^{s+1}\left(X_{1}^{2 d^{\prime}}+\cdots+X_{k}^{2 d^{\prime}}+1\right)$, where $\zeta$ is a new variable, and $2 d^{\prime}$ is any even number greater than $\sum_{1 \leq i \leq s} \operatorname{deg}\left(P_{i}\right)$. Let $S_{\zeta}$ be the set defined by $(Q \geq 0) \wedge_{1 \leq i \leq s}\left((1-\zeta) P_{i}+\zeta \geq 0\right)$. Then, for all sufficiently small $\zeta>0, H_{*}(S)=H_{*}\left(S_{\zeta}\right)$. Moreover, $S_{\zeta}$ is bounded by connected components of a smooth hypersurface $Z(Q)$, which has a finite number of critical points for the projection map onto the $X_{1}$ coordinate and these critical points are nondegenerate and have distinct $X_{1}$ coordinates.

Proof. Let $S_{\zeta}=(Q \geq 0) \wedge_{1 \leq i \leq s}\left((1-\zeta) P_{i}+\zeta \geq 0\right)$. Since $S$ is compact there exists a constant $R$ such that $x \in S \Rightarrow x_{1}^{2 d^{\prime}}+\cdots+x_{k}^{2 d^{\prime}}+1<R$. For $0<\zeta<1 / R$, any point $x=\left(x_{1}, \ldots, x_{k}\right)$ satisfying $P_{1}(x) \geq 0, \ldots, P_{s}(x) \geq 0$ will also satisfy $Q \geq 0$. This follows directly from the definition of $Q$ and the fact that $x_{1}^{2 d^{\prime}}+\cdots+x_{k}^{2 d^{\prime}}+1<R$.

Thus, for every connected component $C$ of $S$ there exists a connected component $C^{\prime}$ of $Q \geq 0$ such that $C \subset C^{\prime}$. Moreover, the signs of the polynomials $\zeta+(1-\zeta) P_{i}$, $1 \leq i \leq s$, cannot change over $C^{\prime}$ because if one of them became zero $Q$ would be negative at that point. However, since $C^{\prime}$ contains $C$, and $\zeta$ is sufficiently small, it is clear that $\zeta+(1-\zeta) P_{i}>0,1 \leq i \leq s$, over $C^{\prime}$. Thus, for $\zeta$ small enough, $S \subset S_{\zeta}$.

Moreover, $S=\bigcap_{t>0} S_{t}$, and $S$ and $S_{t}$ are compact. Thus using Hardt's triviality and Theorem 4, we have that, for small enough $\zeta>0, H_{*}(S) \cong H_{*}\left(S_{\zeta}\right)$.

We next show that the set $S_{\zeta}$ is bounded by connected components of the smooth hypersurface defined by $Q=0$.

First observe that the set $Q \geq 0$ is bounded. This follows from the fact that $2 d^{\prime}>s d$ and thus the second term in $Q$ dominates the first as $|x|$ becomes large.

Secondly, the polynomials $\zeta+(1-\zeta) P_{i}$ are all strictly positive over $S_{\zeta}$. Hence, $S_{\zeta}$ must be bounded by the hypersurface $Z(Q)$.

It remains to show that the hypersurface $Z(Q)$ is smooth and has a finite number of critical points for the projection map onto the $X_{1}$ coordinate, and that these critical points are nondegenerate with distinct $X_{1}$ coordinate.

Let $Q_{t}=\prod_{1 \leq i \leq s}\left(t+(1-t) P_{i}\right)-t^{s+1}\left(X_{1}^{2 d^{\prime}}+\cdots+X_{k}^{2 d^{\prime}}+1\right)$.

Now, $Z\left(Q_{t}\right)$ is smooth if the following system of equation has no solutions over complex projective space:

$$
\bar{Q}_{t}=\frac{\partial \bar{Q}_{t}}{\partial X_{0}}=\cdots=\frac{\partial \bar{Q}_{t}}{\partial X_{k}}=0,
$$

where $\bar{Q}_{t}$ is the homogenization of $Q_{t}$.

The set $T$ of complex $t$ 's for which this system has no solutions in complex projective space is Zariski constructible, open, and contains $t=1$. Hence, it contains $\zeta$ for all sufficiently small real $\zeta>0$, and thus $Z(Q)$ is smooth. 
Also note that, for all $t \neq 0$, the ideal generated by

$$
\left\{Q_{t}, \frac{\partial Q_{t}}{\partial X_{2}}, \ldots, \frac{\partial Q_{t}}{\partial X_{k}}\right\}
$$

is zero-dimensional. This follows from a simple Grobner basis argument which can be found in [3]. Moreover, for $t=1$, all distinct projective solutions over the algebraic closure of the zero-dimensional homogenized system

$$
\bar{Q}_{t}=\frac{\partial \bar{Q}_{t}}{\partial X_{2}}=\cdots=\frac{\partial \bar{Q}_{t}}{\partial X_{k}}=0
$$

have distinct $X_{1}$ coordinates. Moreover, all these critical points are nondegenerate (the Hessian matrix is nonzero at these points).

Again, the set of $t$ 's for which the above conditions are met is Zariski constructible, open, and contains $t=1$, and hence contains $\zeta$ for all sufficiently small $\zeta>0$.

We also need the following proposition.

Proposition 5. Let $Q$ be the same polynomial as in the previous proposition and let $Q_{1}=X_{k+1}^{2 d^{\prime}}-Q$, where $X_{k+1}$ is a new variable.

Let $S_{1} \subset R^{k+1}$ be the set defined by $\left(Q_{1}=0\right) \wedge_{1 \leq i \leq s}\left((1-\zeta) P_{i}+\zeta \geq 0\right)$, let $S_{2} \subset R^{k}$ be the set defined by $(Q=0) \wedge_{1 \leq i \leq s}\left((1-\zeta) P_{i}+\zeta \geq 0\right)$, and let $S_{3} \subset R^{k}$ be the set defined by $(Q \geq 0) \wedge_{1 \leq i \leq s}\left((1-\zeta) P_{i}+\zeta \geq 0\right)$. Then, for all sufficiently small $\zeta>0$, $\chi\left(S_{1}\right)=2 \chi\left(S_{3}\right)-\chi\left(S_{2}\right)$.

Moreover, the connected components of $S_{1}$ are the connected components of a smooth hypersurface $Z\left(Q_{1}\right)$, which has a finite number of critical points for the projection map onto the $X_{1}$ coordinate and these critical points are nondegenerate and have distinct $X_{1}$ coordinates.

Proof. Firstly, note that $Z\left(Q_{1}\right)$ consists topologically of two copies of the set defined by $Q \geq 0$ glued along $Z(Q)$. Moreover, $S_{1}, S_{2}, S_{3}$ are all compact and unions of connected components of the sets defined by $Q_{1}=0, Q=0, Q \geq 0$, respectively. A straightforward application of the Mayer-Vietoris sequence gives $\chi\left(S_{1}\right)=2 \chi\left(S_{3}\right)-\chi\left(S_{2}\right)$.

The proof of the second part of the proposition is entirely similar to the proof of the corresponding properties for $Z(Q)$ given earlier.

We first state the theorem for an algebraic set. This theorem appears in [20], but without a precise bound.

Theorem 5. Let $Z(Q) \subset R^{k}$ be a real algebraic set, and let $m$ be the number of distinct monomials that appear in $Q$. Then the sum of the Betti numbers of $Z(Q)$ is bounded by $2^{O\left(k^{2} m^{4}\right)}$.

Proof. $\quad$ Replace $Z(Q)$ by the set $V$ defined by $Q_{1}=\left(\zeta+(1-\zeta) Q^{2}\right)-\zeta^{2}\left(X_{1}^{2 d}+\cdots+\right.$ $\left.X_{k}^{2 d}+1\right) \geq 0,\left(\zeta+(1-\zeta) Q^{2}\right) \geq 0$ where $2 d>\operatorname{deg}(Q)$, and $\zeta>0$ and sufficiently 
small. Then as in the proof of Proposition 4 we can show that $V$ is bounded by a smooth hypersurface and has a finite number of nondegenerate critical points for the projection map onto the $X_{1}$ coordinates. Moreover, $V$ has the same homology groups as $Z(Q)$. The number of critical points bounds the sum of the Betti numbers of $V$ and hence of $Z(Q)$. Moreover, the number of critical points is bounded by the number of real solutions of the system $Q_{1}=\partial Q_{1} / \partial X_{2}=\cdots=\partial Q_{1} / \partial X_{k}=0$. The total number of nonzero monomials appearing in this system is bounded by $\mathrm{km}^{2}+2 k=O\left(\mathrm{~km}^{2}\right)$.

We can now apply Khovansky's bound on the number of real solutions of a system of $k$ polynomials in $k$ variables [20] to obtain the bound stated in the theorem.

We can now state and prove the theorem in the general case.

Theorem 6. Let $S \subset R^{k}$ be the intersection of a closed semi-algebraic set defined by a quantifier-free Boolean formula without negations involving a family, $\mathcal{P}=\left\{P_{1}, \ldots, P_{s}\right\}$, of $s$ polynomials, whose atoms are of the form $P_{i} \sigma 0, \sigma \in\{\geq, \leq,=\}$, with the zero set $Z(Q)$, of a polynomial $Q$. Let the geometric dimension of $Z(Q)$ be $k^{\prime}$, and let the total number of monomials occurring in the polynomials in $\mathcal{P} \cup\{Q\}$ be bounded by $m$. Then the sum of the Betti numbers of $S$ is bounded by $s^{k^{\prime}} 2^{O\left(k^{2} m^{4}\right)}$.

Secondly, if $S$ is defined by $Q=0, P_{1}>0, \ldots, P_{s}>0$, we have a slightly tighter bound of $\left(\begin{array}{l}s \\ k^{\prime}\end{array}\right) 2^{O\left(k^{2} m^{4}\right)}$.

Proof. The proof is the same as that of Theorem 1 except that in the case of algebraic sets we use the bound in Theorem 5 rather than the Thom-Milnor bound of $O(d)^{k}$.

Note that this bound is again separated into a combinatorial part of $s^{k^{\prime}}$, and an algebraic part which depends only on $m$ and $k$. Also note that the Thom-Milnor method does not give this bound even for the basic semi-algebraic sets. The reason is that in their method one replaces a basic semi-algebraic set by a set defined by a single polynomial which is a perturbed version of their product. However, the number of monomials in the product of $s$ polynomials is exponentially large in $s$, and thus the separation of the combinatorial and the algebraic parts is no longer possible.

The above techniques have been extended recently to prove similar bounds on the Betti numbers of semi-Pffafian sets, with separation of combinatorial and algebraic parts [32].

\section{Computing the Euler Characteristic}

By Proposition 3 we can assume, without loss of generality, that the given semi-algebraic set is compact. If the given set is not compact, then we make the perturbations described in Section 2 and compute the Euler characteristic of the perturbed set. The Euler characteristic of this new set is equal to the Euler characteristic of the original set. The new system will have at most $4 s+1$ polynomials with degrees at most $2 d$. Moreover, we now have to compute in a larger ring $Z[\delta, \varepsilon, \Omega]$. However, since we have introduced only three infinitesimals, the asymptotic complexity of the algorithm is not affected. 


\subsection{Algorithmic Preliminaries}

In our algorithm we utilize several other algorithms from real algebraic geometry as subroutines. In this section we recall some of the algorithms that we use as subroutines, with appropriate pointers to the literature.

We use a subroutine from [3] that constructs univariate representations of the zeros of a zero-dimensional variety. This subroutine takes as input a Gröbner basis of a zerodimensional ideal, $I$, of polynomials in $k$ variables and outputs a set consisting of $(k+2)$ tuples of univariate polynomials $\left(f, g_{0}, \ldots, g_{k}\right)$ such that the complex zeros of $I$ are among the points obtained by evaluating the rational functions $\left(g_{1} / g_{0}, \ldots, g_{k} / g_{0}\right)$ at the roots of the univariate polynomial $f$, for all the tuples $\left(f, g_{0}, \ldots, g_{k}\right)$ in the output. We say that the real points corresponding to the tuple $\left(f, g_{0}, \ldots, g_{k}\right)$ are associated to the tuple, and the tuple itself is a univariate representation of these points. Moreover, if the degrees of the polynomials in the input are bounded by $d$, the degrees of the polynomials in the output as well as the complexity of this subroutine is bounded by $d^{O(k)}$.

We also make use of an algorithm, called the sample points subroutine [3], that computes a finite set of points which intersects every connected component of every nonempty sign condition (referred to as cells henceforth) of a family of polynomials $\mathcal{P}$, of size $s$ and degrees bounded by $d$. The subroutine also outputs the sign vector of the polynomials of $\mathcal{P}$ at each output point. The complexity of this subroutine is $\left(\begin{array}{c}O(s) \\ k\end{array}\right) s d^{O(k)}=(s / k)^{k} s d^{O(k)}$.

Lastly, we make use of a multivariate sign determination subroutine [3]. The input is a system $T$ of polynomial equations in $k$ variables, with a finite number of zeros, along with a Gröbner basis for the ideal generated by the polynomials in $T$, and a set of $s$ polynomials $\mathcal{P}=\left\{P_{1}, \ldots, P_{s}\right\}$. The output is the list of nonempty sign conditions $\sigma_{1}, \ldots, \sigma_{M}$ of $\mathcal{P}$ at the real zeros of the system $T$ and the numbers $c_{1}, \ldots, c_{M}$, where $c_{i}$ is the number of real zeros of $T$ at which the sign vector of $\mathcal{P}$ is $\sigma_{i}$. Moreover, if the polynomials in the input have degrees bounded by $d$, the complexity of this subroutine is $d^{O(k)}$.

We also use this subroutine in the special case of computing the index of a symmetric square matrix of size $k \times k$, with polynomial entries, at the real zeros of a zero-dimensional system. Again, if the degrees of the polynomials in the input are bounded by $d$, the complexity of the subroutine is bounded by $(k d)^{O(k)}$ (see [26]).

\subsection{The Algorithm for a Semi-Algebraic Set Defined by One Sign Condition}

In this section we describe an algorithm for computing the Euler characteristic of a semi-algebraic set defined by one single sign condition on a family of polynomials. Using Lemma 4 we can assume, without loss of generality, that the semi-algebraic set $S$ is defined by $P_{1} \geq 0, \ldots, P_{s} \geq 0$.

From Propositions 5 and 4 it follows that $\chi(S)=\left(\chi\left(S_{1}\right)+\chi\left(S_{2}\right)\right) / 2$. We actually compute $\chi\left(S_{1}\right)$ and $\chi\left(S_{2}\right)$ by computing the indices of the critical points of the projections map to the $X_{1}$ coordinate.

The details are as follows: 
5.2.1. Description of the Algorithm. Given a basic semi-algebraic set $S$ defined by $P_{1} \geq 0, \ldots, P_{s} \geq 0$, where $\operatorname{deg}\left(P_{i}\right)<d$ for $1 \leq i \leq s$, the algorithm computes $\chi(S)$. We first introduce a positive infinitesimal $\zeta$ and construct the following two polynomials:

$$
\begin{gathered}
Q=\prod_{1 \leq i \leq s}\left(\zeta+(1-\zeta) P_{i}\right)-\zeta^{s+1}\left(X_{1}^{2 d^{\prime}}+\cdots+X_{k}^{2 d^{\prime}}+1\right) \\
Q_{1}=X_{k+1}^{2 d^{\prime}}-Q
\end{gathered}
$$

where $X_{k+1}$ is a new variable.

For the following two zero-dimensional systems, $Q=\partial Q / \partial X_{2}=\cdots=\partial Q / \partial X_{k}=$ 0 and $Q_{1}=\partial Q_{1} / \partial X_{2}=\cdots=\partial Q_{1} / \partial X_{k+1}=0$, we first check, using the univariate representation subroutine and for every real zero $p$ of these systems, whether the polynomials $\zeta+(1-\zeta) P_{1}, \ldots, \zeta+(1-\zeta) P_{s}$ are all nonnegative at the point $p$. We retain only those real solutions for which this is satisfied.

For each real zero (critical point) $p$ retained so far for the first system, we compute the index of the critical point, which is the index of the Hessian matrix $\left(\partial^{2} Q / \partial X_{i} \partial X_{j}\right)_{i j}, 2 \leq$ $i, j \leq k$, evaluated at $p$. For $0 \leq i \leq k$ let $c_{i}$ denote the number of critical points of index $i$.

Similarly, for each real zero (critical point) $p$ retained so far for the second system, we compute the index of the critical point, which is the index of the Hessian matrix $\left(\partial^{2} Q_{1} / \partial X_{i} \partial X_{j}\right)_{i j}, 2 \leq i, j \leq k+1$, evaluated at $p$. For $0 \leq i \leq k+1$ let $c_{i}$ denote the number of critical points of index $i$.

We output $\chi(S)=\left(\sum_{0 \leq i \leq k}(-1)^{i} c_{i}+\sum_{0 \leq i \leq k+1}(-1)^{i} d_{i}\right) / 2$.

5.2.2. Proof of Correctness. The proof correctness follows immediately from Propositions 4 and 5 and the standard facts of Morse theory [22].

5.2.3. Complexity of the Algorithm. The polynomials $Q$ and $Q_{1}$ have degrees bounded by $O(s d)$. The cost of computing the indices of the Hessian matrices in the last step dominates the cost. We use the sign determination subroutine in the special case of computing the index of a symmetric square matrix of size $k \times k$, with polynomial entries, at the real zeros of a zero-dimensional system. If the degrees of the polynomials in the input are bounded by $D$, the complexity is bounded by $(k D)^{O(k)}$ (see [26]). Thus the total complexity of this algorithm is bounded by $(k s d)^{O(k)}$.

\subsection{The Case of a General Semi-Algebraic Set}

5.3.1. Description of the Algorithm. Using the algorithm in [3] list all nonempty sign conditions, $\sigma_{1}, \ldots, \sigma_{m}$ of the family $\mathcal{P}$, such that the set $S_{j}$ defined by $\sigma_{j}$ is contained in $S$ for $1 \leq j \leq m$.

For each $\bar{j}, 1 \leq j \leq m$, do the following. Without loss of generality assume that $\sigma_{j}$ is of the form $P_{1}=\cdots=P_{l}=0, P_{l+1}>0, \ldots, P_{s}>0$. Let $U_{j}$ be the set defined by $P_{1}=\cdots=P_{l}=0, P_{l+1} \geq 0, \ldots, P_{s} \geq 0$, and let $V_{j}=U_{j} \cap Z\left(\prod_{l<i \leq s} P_{i}\right)$. 
Using the algorithm described in Section 5.2 for computing the Euler characteristic of basic semi-algebraic sets, compute $\chi\left(U_{j}\right)$ and $\chi\left(V_{j}\right)$.

Output $\chi(S)=\sum_{1 \leq j \leq m}\left(\chi\left(U_{j}\right)-\chi\left(V_{j}\right)\right)$.

\subsubsection{Proof of Correctness. First note that the set $S$ is compact.}

Let $\Sigma$ be the set of nonempty sign conditions on the family of polynomials $\mathcal{P} \cup\{Q\}$, whose realizations are contained in $S$. Also, let $S_{\sigma}$ be the set of points satisfying the sign condition $\sigma$. Thus,

$$
S=\bigcup_{\sigma \in \Sigma} S_{\sigma}
$$

For any set $A$, following [31], we define $\beta_{i}^{\prime}(A)$ to be the rank of the $i$ th homology group $H_{i}(\bar{A}, \partial A)$, where $\bar{A}$ is the closure of $A$ in the topology of $R^{k}$ and $\partial A=\bar{A}-A$. Similarly we define $\chi^{\prime}(A)=\sum_{0 \leq i \leq k}(-1)^{i} \beta_{i}^{\prime}(A)$. If $A$ is compact, then $\beta_{i}^{\prime}(A)=\beta_{i}(A)$ and $\chi^{\prime}(A)=\chi(A)$.

We first prove a preliminary lemma. Recall that a semi-algebraic set is semi-closed if it is the difference of two closed semi-algebraic sets.

Lemma 8. Let $X$ be a semi-algebraic set in $R^{k}$ which is bounded and semiclosed. For some polynomial

$$
f \in R\left[X_{1}, \ldots, X_{k}\right],
$$

let $A=X \cap(f \geq 0)$ and $B=X-A$. Then $\chi^{\prime}(X)=\chi^{\prime}(A)+\chi^{\prime}(B)$.

Proof. The proof is the same as the proof of Lemma 5 in [31] and is reproduced here.

From the exact sequence

$$
\cdots \rightarrow H_{i}(\bar{A} \cup \partial X, \partial X) \rightarrow H_{i}(\bar{X}, \partial X) \rightarrow H_{i}(\bar{X}, \bar{A} \cup \partial X) \rightarrow \cdots,
$$

we have $\chi(\bar{X}, \partial X)=\chi(\bar{X}, \bar{A} \cup \partial X)+\chi(\bar{A} \cup \partial X, \partial X)$. Moreover, $\beta_{i}(\bar{X}, \bar{A} \cup \partial X)=$ $\beta_{i}^{\prime}(B)$ and $\beta_{i}(\bar{A} \cup \partial X, \partial X)=\beta_{i}^{\prime}(A)$ (see [31] for a proof). It follows easily that $\chi^{\prime}(X)=$ $\chi^{\prime}(A)+\chi^{\prime}(B)$.

Next, we prove

Lemma 9. $\chi(S)=\sum_{\sigma \in \Sigma} \chi^{\prime}\left(S_{\sigma}\right)$.

Proof. Note that since $S$ is compact, $\chi^{\prime}(S)=\chi(S)$. Also note that for all $i$, and for all sign conditions $P_{1} \sigma_{1} 0, \ldots, P_{i} \sigma_{i} 0$, on the family of polynomials $\left\{P_{1}, \ldots, P_{i}\right\}$, the set $S \cap\left(P_{1} \sigma_{1} 0\right) \cap \cdots \cap\left(P_{i} \sigma_{i} 0\right)$ is semiclosed. This follows from the fact that $S$ is closed. Now consider the sets $A=S \cap\left(P_{1}>0\right), B=S \cap\left(P_{1}=0\right), C=S \cap\left(P_{1}<0\right)$. Now $S=$ $A \cup B \cup C$. Applying Lemma 8 twice we get that $\chi^{\prime}(X)=\chi^{\prime}(A)+\chi^{\prime}(B)+\chi^{\prime}(C)$. Also, $A, B, C$ are themselves semiclosed. We can continue the argument with the polynomial $P_{2}$ and the sets $X \cap\left(P_{2}>0\right), X \cap\left(P_{2}=0\right), X \cap\left(P_{2}<0\right)$ for $X=A, B, C$, and so on. A simple induction completes the argument. 
It is easy to show, following the notation introduced in the algorithm, that $\beta_{i}^{\prime}\left(S_{j}\right)=$ $\beta_{i}\left(U_{j}, V_{j}\right)$, and thus $\chi^{\prime}\left(S_{j}\right)=\chi\left(U_{j}, V_{j}\right)$. The proof of this appears on p. 621 of [31] and is omitted here.

It follows from the exact sequence

$$
\cdots \rightarrow H_{i}\left(V_{j}\right) \rightarrow H_{i}\left(U_{j}\right) \rightarrow H_{i}\left(U_{j}, V_{j}\right) \rightarrow H_{i-1}\left(V_{j}\right) \rightarrow \cdots,
$$

that $\chi\left(U_{j}, V_{j}\right)=\chi\left(U_{j}\right)-\chi\left(V_{j}\right)$. This in conjunction with Lemma 9 shows that $\chi(S)=$ $\sum_{1 \leq j \leq m}\left(\chi\left(U_{j}\right)-\chi\left(V_{j}\right)\right)$, and this proves the correctness of the algorithm.

5.3.3. Complexity Analysis. The cost of computing all the nonempty sign conditions of the family $\mathcal{P}$ is $s^{k+1} d^{O(k)}$. Moreover, there can be only $\left(\begin{array}{l}s \\ k\end{array}\right)(O(d))^{k}$ such nonempty sign conditions. For each such sign condition included in $S$, we call the algorithm for computing the Euler characteristics of basic semi-algebraic sets twice. The sum of the degrees of the polynomials involved in each such call is $O(s d)$. Thus each call costs $(k s d)^{O(k)}$. Hence, the total complexity of the algorithm is bounded by $(k s d)^{O(k)}$.

The bound in the bit model follows easily once we note that bit sizes of the intermediate values are bounded by $L(s k d)^{O(k)}$.

\section{References}

1. A. I. Barvinok, On the Betti numbers of semi-algebraic sets defined by few quadratic inequalities, Mathematische Zeitschrift, 225 (1997), 231-244.

2. S. Basu, On bounding the Betti numbers and computing the Euler characteristics of semi-algebraic sets, Proc. 28th Annual ACM Symposium on the Theory of Computing, pp. 408-417, (1996).

3. S. Basu, R. Pollack, and M.-F. Roy, On the combinatorial and algebraic complexity of quantifier elimination, Journal of the ACM, 43(6) (1996), 1002-1045.

4. S. Basu, R. Pollack, and M.-F. Roy, On the number of cells defined by a family of polynomials on a variety, Mathematika, 43 (1996), 120-126.

5. S. Basu, R. Pollack, and M.-F. Roy, Computing roadmaps of semi-algebraic sets on a variety (extended abstract), Proc. First Conference on the Foundations of Computational Mathematics, F. Cucker and M. Shub, eds., Springer-Verlag, New York, 1997, pp. 1-15 (full version to appear in the Journal of the AMS).

6. J. Bochnak, M. Coste, and M.-F. Roy, Géométrie algébrique réelle. Springer-Verlag, New York, 1987.

7. J. Canny, Computing road maps in general semi-algebraic sets, Computer Journal 36 (1993), 504-514.

8. J. Canny, D. Grigoriev, and N. Vorobjov, Finding connected components of a semi-algebraic set in subexponential time, Applicable Algebra in Engineering, Communication and Computing, 2 (1992), 217-238.

9. G. E. Collins, Quantifier elimination for real closed fields by cylindrical algebraic decomposition, Lecture Notes in Computer Science, vol. 33, pp. 515-532, Springer-Verlag, Berlin, 1975.

10. S. Eilenberg and N. Steenrod, Foundations of Algebraic Topology, Princeton University Press, Princeton, NJ, 1952.

11. A. Gabrielov, Multiplicities of zeros of polynomials on trajectories of polynomial vector fields and bounds on degree of nonholonomy, Mathematical Research Letters, 2(4) (1995), 437-451.

12. J. E. Goodman and R. Pollack, Upper bounds for configurations and polytopes in $R^{d}$, Discrete and Computational Geometry, 1 (1986), 219-227.

13. J. E. Goodman, R. Pollack, and R. Wenger, Bounding the number of geometric permutations induced by $k$-transversals, Journal of Combinatorial Theory, Series A, 75 (1996), 187-197.

14. L. Gournay and J. J. Risler, Construction of roadmaps of semi-algebraic sets, Technical Report, DMI, Ecole Normale Superieure, Paris, (1992).

15. D. Grigoriev, N. Vorobjov, and J. Canny, Counting connected components of a semi-algebraic set in sub-exponential time, Computational Complexity, 2(2) (1992), 133-186. 
16. R. M. Hardt, Semi-algebraic local triviality in semi-algebraic mappings, American Journal of Mathematics, 102 (1980), 291-302.

17. J. Heintz, T. Recio, and M.-F. Roy, Algorithms in real algebraic geometry and applications to computational geometry, Discrete and Computational Geometry: Selected Papers from the DIMACS Special Year, J. E. Goodman, R. Pollack, and W. Steiger, eds. AMS, Providence, RI, and ACM, New York, 1991, vol. 6, pp. 137-164.

18. J. Heintz, M.-F. Roy, and P. Solernò, Single exponential path finding in semi-algebraic sets, II: The general case, in Algebraic Geometry and Its Applications, C. L. Bajaj, ed., Springer-Verlag, New York, 1993, pp. 467-481.

19. J. Heintz, M.-F. Roy, and P. Solernò, Description of the connected components of a semi-algebraic set in single exponential time, Discrete and Computational Geometry, 11 (1994), 121-140.

20. A. G. Khovansky, Fewnomials, Translations of Mathematical Monographs, Vol. 88, AMS, Providence, RI, 1991.

21. M. Knebusch, Semi-algebraic topology in the last ten years, in Real Algebraic Geometry, M. Coste, L. Mahe, and M.-F. Roy, eds., Lecture Notes in Mathematics, vol. 1524, pp. 1-36, Springer-Verlag, Berlin, 1992.

22. J. Milnor, Morse Theory, Annals of Mathematical Studies, Princeton University Press, Princeton, NJ, 1963.

23. J. Milnor, On the Betti numbers of real varieties, Proceedings of the AMS 15 (1964), 275-280.

24. J. L. Montana, J. E. Morais, and L. M. Pardo, Lower bounds for arithmetic networks II: Sum of Betti numbers, Applicable Algebra in Engineering, Communication and Computing, 7(1) (1996), 41-51.

25. O. A. Oleinik and I. B. Petrovsky, On the topology of real algebraic surfaces, Izvestiya Akademii Nauk SSSR 13 (1949), 389-402.

26. P. Pedersen, M.-F. Roy, and A. Szpirglas, Counting real zeros in the multivariate case, in Computational Algebraic Geometry, F. Eyssette and A. Galligo, eds. Progress in Mathematics, vol. 109, pp. 203-224, Birkhauser, Basel, 1993.

27. J. Schwartz and M. Sharir, On the "piano movers" problem, II. General techniques for computing topological properties of real algebraic manifolds, Advances in Applied Mathematics, 4 (1983), 298-351.

28. E. H. Spanier, Algebraic Topology, McGraw-Hill, New York, 1966.

29. R. Thom, Sur l'homologie des varietes algebriques reelles, in Differential and Combinatorial Topology, S. S. Cairns, ed., Princeton University Press, Princeton, NJ, 1965, pp. 255-265.

30. H. Warren, Lower bounds for approximation by nonlinear manifolds, Transactions of the AMS, 133 (1968), 167-178.

31. A. C. Yao, Decision tree complexity and Betti numbers, Proc. 26th ACM Symposium on Theory of Computing, pp. 615-624, 1994.

32. T. Zell, Betti numbers of semi-Pfaffian sets, Preprint.

Received September 9, 1997, and in revised form March 18, 1998, and October 5, 1998. 